\title{
The reductive activity of human liver microsomes for vitamin $K$ epoxides
}

\author{
Masashi Kawano ${ }^{1,2}$, Takuya Araki ${ }^{12^{*}}$, Hideaki Yashima ${ }^{1}$, Tomonori Nakamura ${ }^{3}$, Koujirou \\ Yamamoto ${ }^{1,2}$
}

1. Department of Pharmacy, Gunma University Hospital, 3-39-15 Showa-machi, Maebashi, Gunma 3718511, Japan

2. Department of Clinical Pharmacology and Therapeutics, Gunma University Graduate School of Medicine, 3-39-22 Showa-machi, Maebbashi, Gunma 371-8511, Japan

3. Faculty of Pharmacy, Keio University, 1-5-30 Shibakoen, Minato-ku, Tokyo 105-8512, Japan

Received: 27 Dec 2019/Revised: 3 Jan 2020/Accepted: 13 Jan 2020/Published: 13 Feb 2020

\begin{abstract}
Vitamin $\mathrm{K}(\mathrm{VK})$ is oxidized to vitamin $\mathrm{K}$ epoxide (VK-O) during the production of VK-dependent blood clotting factors. Thereafter, VK-O is reduced to VK by vitamin $\mathrm{K}$ epoxide reductase (VKOR) in the liver and reused. This reductive reaction is inhibited by warfarin, an oral anticoagulant. VK in nature is roughly divided into two types, VK1 (phylloquinone) and VK2 (menaquinone). Although their bioavailabilities and elimination half-lives from human blood differ, information on the influence of each VK on the effectiveness of warfarin is limited. In this study, the difference in the metabolism of VK1-O and MK4-O by VKOR was evaluated in an in vitro study using human liver microsomes. The results showed that the substrate affinity $(1 / \mathrm{Km})$, and the maximum reaction rate (Vmax) of the VKOR reduction was around 7 and 4 times higher for MK4-O than for VK1-O, respectively. The intrinsic clearance of MK4-O, obtained by dividing the Vmax value by the Km value, was about 30 times greater than that of VK1-O. According to these data, the production of VK-dependent blood coagulation factors can be considered to be dominated mainly by MK4-O, at least under normal conditions. We may thus have to be more careful about controlling the intake of MK4 than VK1 in patients receiving warfarin therapy.
\end{abstract}

Keywords: vitamin K1 (phylloquinone), vitamin K2 (menaquinone), VKOR, warfarin

\section{Introduction}

Vitamin $\mathrm{K}(\mathrm{VK})$ is essential for the production of VK-dependent blood clotting factors (blood clotting factors II, VII, IX, and X) during blood clotting. Subsequently, VK is oxidized to vitamin $\mathrm{K}$ epoxide (VK-O). Thereafter, $\mathrm{VK}-\mathrm{O}$ is reduced to $\mathrm{VK}$ by vitamin $\mathrm{K}$ epoxide reductase (VKOR) in the liver and reused [1].

Warfarin, one of the most widely used oral anticoagulants, inhibits VKOR, depletes VK, and reduces the production of coagulation factors $[2,3]$. Because of its high effectiveness, warfarin is used for the treatment and prevention of thromboembolism in various pathologies associated with increased blood coagulation ability, such as venous thromboembolism, atrial fibrillation, and prosthetic valve replacement [4,5]. On the other hand, the efficacy of warfarin varies widely among individuals, with multiple genetic variations

${ }^{*}$ Corresponding author,

e-mail : tkyaraki@gunma-u.ac.jp (T. Araki) identified as causative factors [6,7]. However, genetics cannot fully explain individual variations, and the influence of dietary VK intake is considered one of the other major factors besides genetic factors [8-10]. Because warfarin inhibits the reuse of VK by acting on VKOR, its effect is attenuated by the ingestion of drugs and foods containing large amounts of VK. These interactions are clinically important, and many VK-containing foods such as broccoli, chlorella, Japanese traditional foods, nori (seaweed) and natto (fermented soybean), and pig liver have been reported to potentially attenuate the efficacy of warfarin [11-15].

$\mathrm{VK}$ is a general term for a number of substances with 2-methyl-1,4-naphthoquinone (VK3) as a common structure. Structurally, these substances have side chains of different lengths and degrees of unsaturation bonded to the carbon at position 3 . VK in nature is roughly divided into two types, 
VK1 and VK2. VK1 is contained in plant foods such as broccoli, where it is known as phylloquinone. VK1 thus comprises the majority of human VK intake and is generally considered to account for more than $90 \%$ of total VK intake [16]. On the other hand, VK2, which is also called menaquinone (MK), is mainly present in the liver of mammals and comprises a group of 14 substances (MK1 to MK14) that differ in the number of isoprene units constituting the prenyl side chain [17]. Two forms of MK consumed by humans are MK4, mainly found in chicken and egg yolk, and MK7, produced mainly by nattokinase and found in natto. Human intestinal bacteria synthesize MK10 and MK11 and, to a lesser extent, MK7, MK8, MK9, and MK12 [18]. However, because the amount of MK synthesized by enterobacteria is minuscule compared to the VK requirement, dietary intake of VK is considered essential [19]. The absorption rate from the intestinal tract and elimination halflife from human blood differ between VK1 and VK2 [20]. In addition, VK1 is metabolized to VK3 during the small intestine absorption process and then converted to MK4 in peripheral tissues [21,22]. However, detailed information on the influence of each VK on the effectiveness of warfarin is lacking.

In this study, in order to clarify the differences in the effects of VK1 and VK2 on VKdependent blood coagulation factor generation, the VKOR metabolic activities for VK1-O and MK4-O were evaluated in an in vitro study using human liver microsomes (HLMs).

\section{Methods}

\subsection{Materials}

$\mathrm{AgNO}_{3}, \quad$ dithiothreitol (DTT), Tris(hydroxymethyl)aminomethane, and MK4 were purchased from FUJIFILM Wako Pure Chemical Corporation (Osaka, Japan), whereas phylloquinone (VK1), phylloquinone epoxide (VK1-O), and menaquinone-4 epoxide (MK4-O) were purchased from Sigma Aldrich Japan (Osaka, Japan). MK5 was generously supplied by Eizai Co., Ltd. (Tokyo, Japan). Pooled HLMs (Lot \#. 88114) were purchased from Nippon Becton Dickinson Co., Ltd. (Tokyo, Japan). All other reagents were obtained from commercial sources and were
HPLC-grade or special-grade reagents.

VK1, MK4, MK5, VK1-O, and MK4-O were stored at $-20^{\circ} \mathrm{C}$ under dark conditions. Stock solutions of VK1, MK4, and MK5, were prepared by dissolving each compound in ethanol at $10 \mathrm{mg} /$ $\mathrm{mL}$ and then diluted in methanol to the appropriate concentration before use. Stock solutions of VK1-O and MK4-O were prepared by dissolving each compound in isopropanol at $10 \mathrm{mg} / \mathrm{mL}$ and then diluted in methanol to the appropriate concentration before use. All stock solutions were stored at $4^{\circ} \mathrm{C}$ under dark conditions. HLMs were stored at $-80^{\circ} \mathrm{C}$ and diluted in water to the appropriate concentration before use.

\subsection{Enzymatic reduction of VK-O by HLMs}

The reaction mixture was prepared by mixing $35 \mu \mathrm{L}$ distilled water, $2.5 \mu \mathrm{L}$ methanol, $25 \mu \mathrm{L}$ HLMs (final concentrations 0.1 and $0.01 \mathrm{mg} / \mathrm{mL}$ for the assay of VK1-O and MK4-O, respectively), $180 \mu \mathrm{L}$ reaction buffer, and $2.5 \mu \mathrm{L}$ of substrate (VK1-O or MK4-O solution). The reaction buffer comprised a 2:1:1 mixture of $60 \%$ glycerol:100 $\mathrm{mM}$ Tris(hydroxymethyl)aminomethane $\quad(\mathrm{pH}$ 8.6):0.6 $\mathrm{M} \mathrm{NaCl}$.

The reaction mixture was incubated at $37^{\circ} \mathrm{C}$ for 5 min using a block incubator as preincubation and the reaction was started by the addition of $5 \mu \mathrm{L}$ of $200 \mathrm{mM}$ DTT. After $10 \mathrm{~min}$ at $37^{\circ} \mathrm{C}, 500 \mu \mathrm{L}$ of icecold $0.05 \mathrm{M} \mathrm{AgNO}_{3}$ :isopropanol (5:9) solution was added to stop the reaction. MK5 $(500 \mu \mathrm{L})$ was used as an analytical internal standard. Next, $500 \mu \mathrm{L}$ of $\mathrm{n}$-hexane was added, the samples were vigorously shaken for 15 min with a mechanical shaker, and the mixture was centrifuged at 13,000 rpm for $5 \mathrm{~min}$. The upper organic layer was transferred to another polyethylene tube, and evaporated to dryness under reduced pressure and dark conditions. The residue was dissolved in $100 \mu \mathrm{L}$ of methanol, and $10 \mu \mathrm{L}$ of the solution was used for LC-MS/MS analysis.

The HLM concentration, reaction time and initial concentration of substrates were determined according to the results of a preliminary study. Briefly, VK1 production increased with an 
increasing HLM concentration in a final HLM concentration range of $0.05-0.2 \mathrm{mg} / \mathrm{mL}$ when the initial concentration of $\mathrm{VK} 1-\mathrm{O}$ and the reaction time were fixed at $1 \mu \mathrm{M}$ and $10 \mathrm{~min}$, respectively. For MK4, MK4 production increased with an increasing HLM concentration in a final HLM concentration range of $0.005-0.02 \mathrm{mg} / \mathrm{mL}$ when the initial concentration of MK4-O and the reaction time were fixed at $0.01 \mu \mathrm{M}$ and $10 \mathrm{~min}$, respectively. Therefore, the final concentrations of HLM in the VK1-O and MK4-O metabolic studies were set at 0.1 and $0.01 \mathrm{mg} / \mathrm{mL}$, respectively (Fig. $1 \mathrm{~A}, \mathrm{C}$ ). In addition, VK1 production increased with an increasing reaction time in the range of 5-15 $\mathrm{min}$ when the initial concentration of VK1-O and the final HLM concentration were fixed at $1 \mu \mathrm{M}$ and 0.1 $\mathrm{mg} / \mathrm{mL}$, respectively. MK4 production increased with an increasing reaction time in a range of 5-15 $\mathrm{mg} / \mathrm{mL}$ when the initial concentration of MK4-O and the final concentration of HLM were fixed at $0.1 \mu \mathrm{M}$ and $0.01 \mathrm{mg} / \mathrm{mL}$, respectively. Therefore, the reaction time for both the VK1-O and MK4-O metabolic studies was set at 10 min (Fig. $1 \mathrm{~B}, \mathrm{D})$. The initial substrate concentration was set to ranges including concentrations to obtain both of the maximum reaction rate and $1 / 2$ of the maximum reaction rate.

\subsection{Enzyme kinetic analysis}

The reaction rate was obtained by measuring the amount of VK1 or MK4 produced from VK1-O (final concentration $1,5,10,50$, or $100 \mu \mathrm{M}$ ) or MK4-O (final concentration 0.1, 0.5, 1, 5, 10, or $50 \mu \mathrm{M})$ as substrates. The reaction rate parameter was calculated by applying the Michaelis-Menten

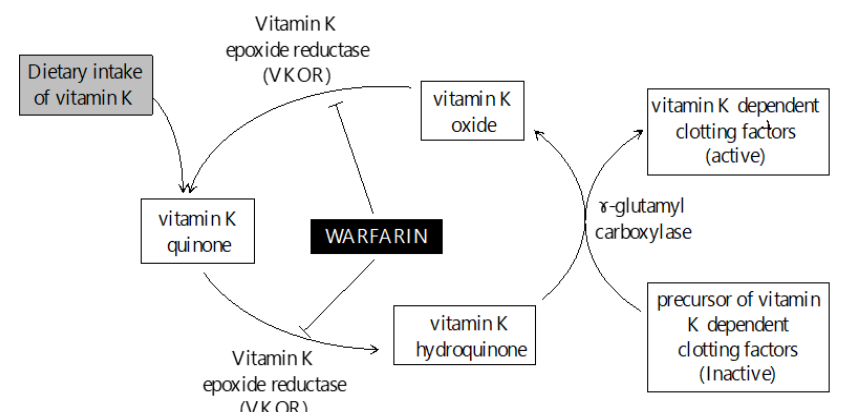

Figure 1. Vitamin K cycle and its inhibition by warfarin equation (Eq. 1) to the relationship between the substrate concentration and the reaction rate.

$\mathrm{v}=\mathrm{V} \max \times[\mathrm{S}] /(\mathrm{Km}+[\mathrm{S}])$, Eq. 1

where v, Vmax, [S], and $\mathrm{Km}$ represent the enzymatic reaction rate, maximum enzymatic reaction rate, concentration of substrate, and Michaelis constant, respectively. For the fitting, the nonlinear least squares method was used. The liver-specific clearance (CLint) was calculated by Equation 2.
CLint $=\mathrm{Vmax} / \mathrm{Km}$
Eq. 2

\subsection{Analysis of $V K s$ and $V K-O s$}

Tandem quadrupole MS was used to analyze VK concentrations. Xevo TQ (Waters, Milford, MA) with ESI spray in positive ionization mode was used with the following ionization parameters: capillary voltage, $1000 \mathrm{~V}$; desolvation temperature, $500^{\circ} \mathrm{C}$; source temperature, $150^{\circ} \mathrm{C}$; desolvation gas flow, $950 \mathrm{~L} / \mathrm{h}$; and cone gas flow, $50 \mathrm{~L} / \mathrm{h}$. The following transitions were monitored: 451/187 for VK1, 467/161 for VK1-O, 445/187 for MK4, 461/121 for MK4-O, and 513/187 for MK5. Sample cone voltages and collision energies were respectively $34 \mathrm{~V}$ and $28 \mathrm{~V}$ for $\mathrm{VK} 1,24 \mathrm{~V}$ and 16 $\mathrm{V}$ for $\mathrm{VK} 1-\mathrm{O}, 22 \mathrm{~V}$ and $22 \mathrm{~V}$ for MK4, $24 \mathrm{~V}$ and $36 \mathrm{~V}$ for MK4-O, and $26 \mathrm{~V}$ and $28 \mathrm{~V}$ for MK5. For separation analysis, LC was performed with an ACQUITY UPLC ${ }^{\circledR}$ system (Waters). An ACQUITY UPLC $^{\circledR}$ BEH C18 column $(2.1 \mathrm{~mm} \times 50 \mathrm{~mm}, 1.7$ $\mu \mathrm{m})$ (Waters) was used as the LC column. The LC conditions were as follows: column temperature, $60^{\circ} \mathrm{C}$; mobile phase, $0.1 \%$ formic acid in $93 \%$ methanol; and flow rate, $0.4 \mathrm{~mL} / \mathrm{min}$. The retention times of VK1, VK1-O, MK4, MK4-O, and MK5 were $2.97,2.30,1.60,1.26$, and 2.75 , respectively. Good linearity was found within the ranges of 5-1000 ng/mL and $0.5-50 \mathrm{ng} / \mathrm{mL}$ for VK1 and MK4, respectively. Intra- and inter-assay precision were under $15 \%$ and the accuracy were over $85 \%$.

\section{Results}

The Michaelis-Menten plot of the reductive reaction of VK1-O and MK4-O by the HLMs is 
shown in Fig. 2 and the estimated MichaelisMenten parameter is listed in Table 1. The Km value of MK4-O was around 7 times lower than that of VK1-O and its Vmax value was around 4 times higher than that of VK1-O. The intrinsic clearance - obtained by dividing the Vmax value by the Km value - was about 30 times greater for MK4-O than for VK1-O.

Table 1. Enzyme kinetic parameters of VK-O reduction

\begin{tabular}{lcc}
\hline & VK1-O & MK4-O \\
\hline $\mathrm{Km}(\mu \mathrm{M})$ & $1.38 \pm 0.37$ & $0.201 \pm 0.032$ \\
$\mathrm{Vmax}(\mathrm{pmol} / \mathrm{min} / \mathrm{mg}$ & $202.5 \pm 9.11$ & $819.2 \pm 22.0$
\end{tabular}
protein)

$\mathrm{CL}_{\text {int }}(\mathrm{mL} / \mathrm{min} / \mathrm{mg}$ protein $)$

0.147

4.069

The data of $\mathrm{Km}$ and Vmax are expressed as mean \pm standard deviation.

Although about $25 \%$ and about $12 \%$ of MK4-O were reduced to MK4 after a 10-min reaction when 0.1 and $0.5 \mu \mathrm{M}$ MK4-O were used as substrate, respectively, the amount of $\mathrm{VK} 1$ and $\mathrm{MK} 4$ produced was less than $10 \%$ of the substrate in the VK1-O and MK4-O metabolic studies at other

A

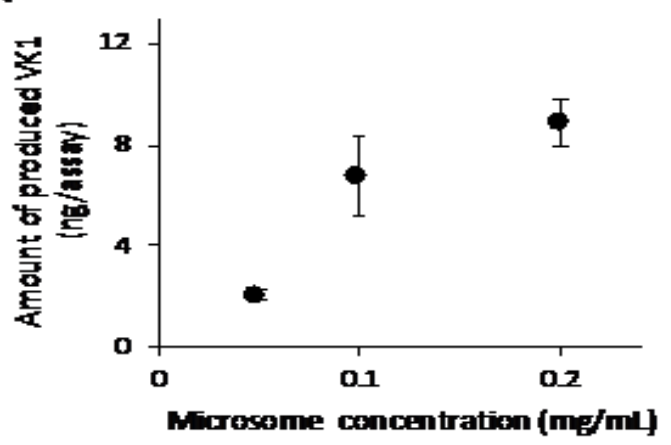

C

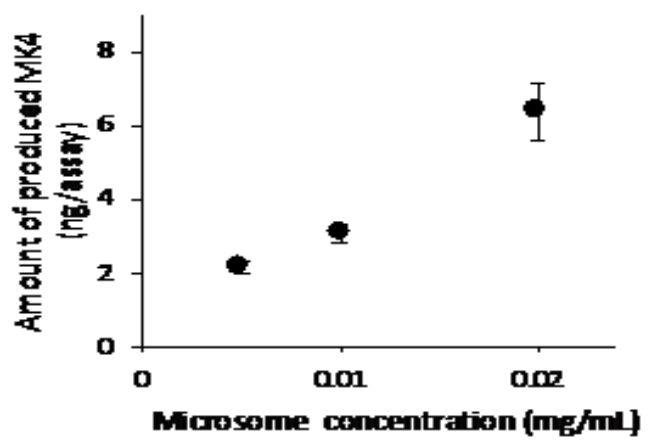

concentrations (table 2).

Table 2. Ratio of metabolized VK-Os to reductants

\begin{tabular}{|c|c|c|}
\hline \multirow{2}{*}{$\begin{array}{c}\text { Substrate } \\
\text { concentration }(\boldsymbol{\mu M})\end{array}$} & \multicolumn{2}{|c|}{ Reduction ratio (\%) } \\
\cline { 2 - 3 } & VK1-O & MK4-O \\
\hline 0.1 & No data & $26.17 \pm 5.57$ \\
\hline 0.5 & No data & $12.03 \pm 2.96$ \\
\hline 1 & $7.00 \pm 1.35$ & $6.76 \pm 1.09$ \\
\hline 5 & $3.46 \pm 0.13$ & $1.56 \pm 0.13$ \\
\hline 10 & $1.93 \pm 0.17$ & $0.79 \pm 0.11$ \\
\hline 50 & $0.38 \pm 0.04$ & $0.17 \pm 0.02$ \\
\hline 100 & $0.19 \pm 0.02$ & No data \\
\hline
\end{tabular}

The reduction ratios are shown as the ratio of VKs produced to their complete reduction. The data are expressed as mean \pm standard deviation.

\section{Discussion}

VK is one of the fat-soluble vitamins essential for the activation of VK-dependent proteins and is involved not only in the production of blood clotting factors, but also in the functional control of the VK-dependent $\gamma$-carboxylate (Gla) protein present in bone, cartilage, vascular smooth muscle, brain/nerve tissue, and kidney $[23,24]$. As described

\section{B}

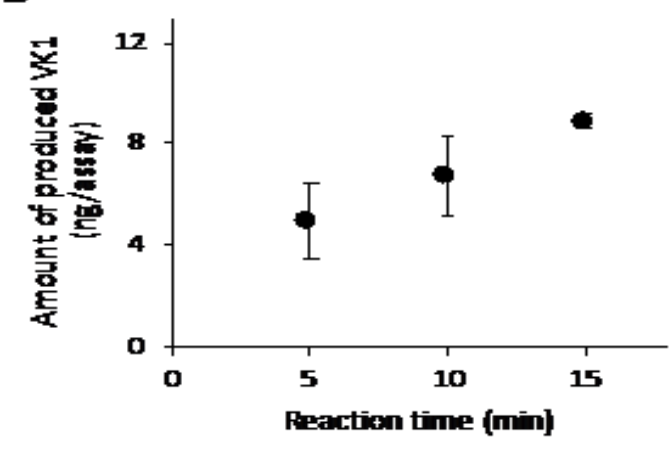

D

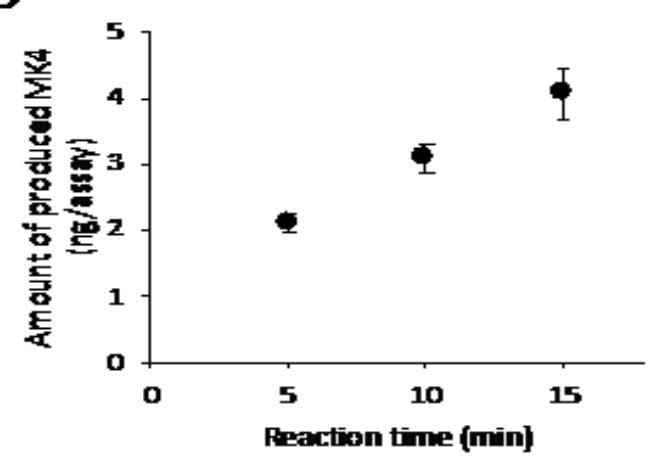

Figure 2. Basic experimental data for determining the experimental conditions. The symbols and error bars represent the mean data and standard deviations, respectively. 


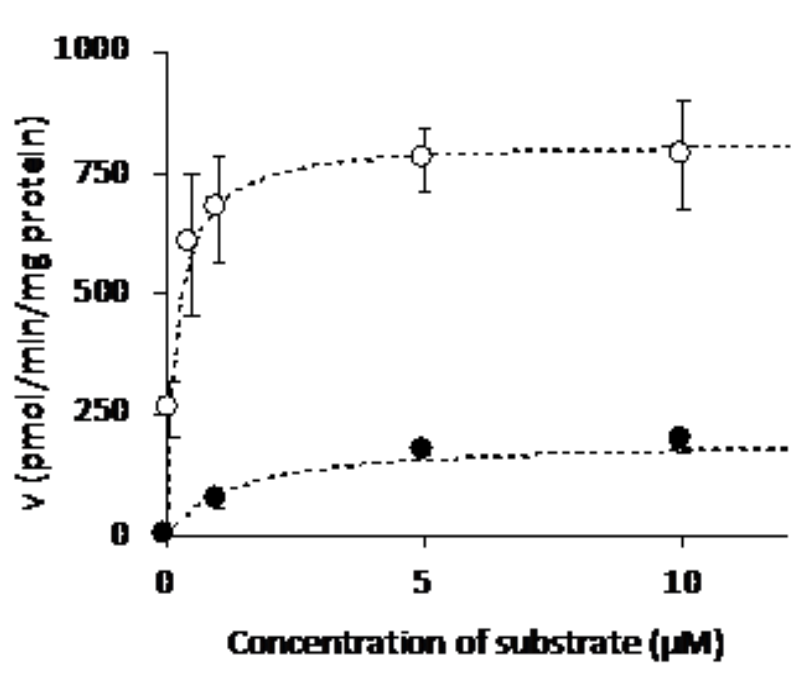

Figure 3. Reduction rate of VK-O by HLMs. The symbols, error bars, and dotted lines represent the mean data, standard deviations, and curve fitting, respectively. Closed and opened circles show VK1 epoxide and MK4 epoxide, respectively.

above, VK is roughly divided into two types-VK1 and VK2 - with markedly different bioavailabilities and in vivo elimination half-lives [20].

In our study, the substrate affinity shown as 1/ $\mathrm{Km}$ and maximum reaction rate shown as Vmax of the MK4-O reduction by VKOR were around 7 and 4 times larger than that of VK1-O reduction. Accordingly, the CLint value of the MK4-O reduction by VKOR was around 30 times larger than that of the VK1-O reduction (Fig. 2, Table 1). Although about $90 \%$ of human VK intake is VK1 [16], the blood concentrations of VK1 and MK4 are similar $(0.044-1.357$ and $0.074-0.759 \mathrm{ng} / \mathrm{mL}$ for VK1 and MK4, respectively) [25], and most of the VK in organs such as the brain, pancreas, and liver are also MK4 [26]. Those data also support our results. In addition, the concentration of VKs and VK-Os in human are known to be much lower level than the that used in our study. So, the reaction of VKOR in body is considered to be not saturated, and the contribution of VK-Os as substrates should depend on the affinities of VKOR to VK-Os. Thus, in a general VK intake situation, the influence of MK4 intake should be greater than that of VK1. Because the physiological substrate of VKOR in mammal liver is reported to be MK4-O, the reuse of VK1, which is not the original substrate, is likely to be inefficient.

The interaction between warfarin and the VK1 found mainly in vegetables and seaweed has been frequently reported $(19,20)$, and the effect of food containing VK1 on the effectiveness of warfarin is transient, disappearing within a few days in many cases $[11,16,27]$.

This phenomenon is thought to be due to the following effects: when a huge amount of VK1 is consumed, most of the VK1, which would activate VK-dependent blood clotting factors, disappears from the body without reuse by VKOR reduction. On the other hand, there are limited reports on the interaction between warfarin and food containing a large amount of VK2. In contrast to the short-term effect of VK1 on the effectiveness of warfarin, the effect of VK2 intake on the activity of warfarin in individuals who ate VK2-containing foods was reported to last for several weeks $[27,28]$. This phenomenon can be explained by the efficient reduction and reuse of MK4 by VKOR. The difference in the interaction between warfarin and VKs is also consistent with our results.

The substrate affinity and maximum reaction rate of the VKOR reduction were somewhat larger for MK4-O than for VK1, and the CLint of VKOR was around 30 times higher for MK4-O than for VK1-O. Therefore, under normal dietary intake conditions, the production of VK-dependent blood clotting factors is considered to be affected mainly by MK4-O, suggesting that individual differences in the effectiveness of warfarin can be suppressed by controlling the intake of MK4. However, because we did not conduct a metabolic study on the coexistence of VK1-O and MK4-O or an interaction study between warfarin and VK1-O or MK4-O, the effects of a large intake of VK1 or MK4 on the effectiveness of warfarin were not clarified. Thus, to elucidate the effect of VK intake on the effectiveness of warfarin, we need to conduct a metabolic study of the coexistence of VK1-O and MK4-O, an interaction study between warfarin and VK1-O or MK4-O, and a clinical study of the blood 
concentration of VKs in patients taking warfarin.

\section{Conclusion}

In conclusion, the substrate affinity and maximum reaction rate of reduction by VKOR are much larger for MK4-O than for VK1, and the CLint of VKOR is around 30 times higher for MK4-O than for VK1-O. These data suggest that individual differences in the effectiveness of warfarin can be suppressed by controlling the intake of MK4.

\section{Acknowledgement}

This study was supported by a grant-in-aid for scientific research KAKENHI 21590150 from Japan Society for the Promotion of Science. Koujirou Yamamoto has received a donation from Eisai Co., Ltd. (Tokyo, Japan). All other authors have no conflict of interest to declare.

\section{References}

[1] Wu S, Chen X, Jin D, Stafford D, Pedersen L, Tie J. Warfarin and vitamin $\mathrm{K}$ epoxide reductase: a molecular accounting for observed inhibition. Blood. 2018;132(6):64757.

[2] Czogalla K, Liphardt K, Honing K, Hornug V, Biswas A, Watzka M, et al. VKORC1 and VKORC1L1 have distinctly different oral anticoagulant dose-response characteristics and binding sites. Blood Adv. 2018;2(6):691702.

[3] Haque J, McDonald M, Kulman J, Rettie A. A cellular system for quantitation of vitamin $\mathrm{K}$ cycle activity: structure-activity effects on vitamin $\mathrm{K}$ antagonism by warfarin metabolites. Blood. 2014;123(4):582-9.

[4] Chan Y, Lee H, See L, Tu H, Chao T, Yeh $\mathrm{Y}$, et al. Effectiveness and safety of four direct oral anticoagulants in Asian patients with nonvalvular atrial fibrillation. Chest. 2019;156(3):529-43.

[5] Lutsey P, Walker R, MacLehouse R, Alonso A, Adam T, Zakai N. Direct oral anticoagulants and warfarin for venous thromboembolism treatment: Trends from 2012 to 2017. Res Pract Thromb Haemost. 2019;3(4):668-73.
[6] Farzamikia N, Sakhinia E, Afrasiabirad A. Pharmacogenetics-based warfarin dosing in patients with cardiac valve replacement: The effects of CYP2C9 and VKORC1 gene polymorphisms. Lab Med. 2017;49(1):25-34.

[7] Zhang J, Klein K, Jorgensen A, Francis B, Alfirevic A, Bourgeois S. Effect of genetic variability in the CYP4F2, CYP4F11, and CYP4F12 genes on liver mRNA levels and warfarin response. Front Phamacol. 2017;8:323.

[8] Liang R, Wang C, Zhao H, Huang J, Hu D, Sun Y. Influence of CYP4F2 genotype on warfarin dose requirement-a systematic review and meta-analysis. Thrombosis Res. 2012;130(1):38-44.

[9] Yang L, Ge W, Yu F, Zhu H. Impact of VKORC1 gene polymorphism on interindividual and interethnic warfarin dosage requirement-a systematic review and meta analysis. Thrombosis Res. 2010;125(4):e159-66.

[10] Al-Eitan L, Almasri A, Khasawneh R. Effects of CYP2C9 and VKORC1 polymorphisms on warfarin sensitivity and responsiveness during the stabilization phase of therapy. Saudi Pharm J. 2019;27(4):484-90.

[11] Leblanc C, Dube M, Presse N, Dumas S, Nguyen M, Rouleau-Milloux E, et al. Avoidance of Vitamin K-Rich Foods Is Common among Warfarin Users and Translates into Lower Usual Vitamin K Intakes. L Acad Nutr Diet. 2016;116(6):1000-7.

[12] Homma K, Wakana N, Suzuki Y, Nukui M, Daimatsu T, Tanaka E, et al. Treatment of natto, a fermented soybean preparation, to prevent excessive plasma vitamin $\mathrm{K}$ concentrations in patients taking warfarin. J Nutr Sci Vitaminol. 2006;52(5):297-301.

[13] Franco V, Polanczyk CA, Clausell N, Rohde LE. Role of Dietary Vitamin K Intake in Chronic Oral Anticoagulation: Prospective Evidence from Observational and Randomized protocols. Am J Med. 2004;116:651-6.

[14] Karlson B, Leijd B, Hellstrom K. On the influence of vitamin K-rich vegetables and wine on the effectiveness of warfarin treatment. Acta Med Scand. 1986;220:34750. 
[15] Chow WH, Chow TC, Tse TM, Tai YT, Lee WT. Anticoagulant instability with life-threating complication after dietary modification. Postgrad Med J. 1990;66:855-7.

[16] Holmes M, Hunt B, Shearer M. The role of dietary vitamin $\mathrm{K}$ in the management of oral vitamin $\mathrm{K}$ antagonists. Blood Rev. 2012;26(1):1-14.

[17] Ferland G, Doucet I, Mainville D. Phylloquinone and menaquinone 4 tissue distribution at different life stages in male and female sprague-dawley rats fed different VK levels since weaning or subjected to a $40 \%$ calorie restriction since adulthood. Nutrients. 2016;8(3):141.

[18] Fu X, Harshman S, Shen X, Haytowitz D, Karl J, Wolfe B, et al. Multiple Vitamin K Forms Exist in Dairy Foods. Curr Dev Nutr. 2017;1(6): e000638

[19] Flore R, Ponziani F, Rienzo T, Zocco M, FlexA, Gerardino L, et al. Something more to say about calcium homeostasis: the role of vitamin $\mathrm{K} 2$ in vascular calcification and osteoporosis. Eur Rev Med Pharmacol. 2013;17(18):2433-40.

[20] Beaulens J, Booth S, van den Heuvel E, Stoecklin E, Vermeer C. The role menaquinones (vitamin K2) in human health. Br J Nutr. 2013;110(8):1357-68.

[21] Hirota Y, Tsugawa N, Nakagawa K, Suhara Y, Tanaka K, Uchino Y, et al. Menadione (vitamin K3) is a catabolic product of oral phylloquinone (vitamin K1) in the intestine and a circulating precursor of tissue menaquinone-4 (vitamin K2) in rats. J Biol Chem. 2013;288(46):33071-80.
[22] Shearer M, Okano T. Key Pathways and Regulators of Vitamin K Function and Intermediary Metabolism. 2018;38:127-51.

[23] Boraldi F, Garci-Fernandez M, PaolinelliDevincenzi C, Annovi G, Schurgers L, Vermeer $\mathrm{C}$, et al. Ectopic calcification in $\beta$-thalassemia patients is associated with increased oxidative stress and lower MGP carboxylation. Biochim Biophys Acta. 2013;1832(12):2077-84.

[24] Mayer O, Seidlerova J, Bruthans J, Filipovsky J, Timoracka K, Vanek J, et al. Desphospho-uncarboxylated matrix Glaprotein is associated with mortality risk in patients with chronic stable vascular disease. Atherosclerosis. 2014;235(1):162-8.

[25] Dunovska K, Klapkova E, Sopko B, CepovaJ, Prusa R. LC-MS/MS quantitative analysis of phylloquinone, menaquinone-4 and menaquinone-7 in the human serum of a healthy population. PeerJ. 2019;7:e7695.

[26] Al Rajab A, Booth S, Peterson J, Choi S, Suttie J, Shea M, et al. Deuterium-labeled phylloquinone has tissue-specific conversion to menaquinone-4 among Fischer 344 male rats. J Nutr. 2012;142(5):841-5.

[27] Violi F, Lip G, Pignateli P, Pastori D. Interaction between dietary vitamin $\mathrm{K}$ intake and anticoagulation by vitamin $\mathrm{K}$ antagonists: Is it really true?: A systematic review. Medicine. 2016;95(10):e2895.

[28] McCabe K, Booth S, Fu X, Shobeiri N, Pang J, Adams M, et al. Dietary vitamin K and therapeutic warfarin alter the susceptibility to vascular calcification in experimental chronic kidney disease. Kidney Int. 2013;83(5):83544. 